\title{
Human Gait Modeling: Dealing with Holonomic Constraints
}

\author{
Tingshu $\mathrm{Hu}^{1 \dagger} \quad$ Zongli Lin ${ }^{1 \dagger} \quad$ Mark F. Abel $^{2 \dagger} \quad$ Paul E. Allaire ${ }^{3 \dagger}$
}

\begin{abstract}
Dynamical models are fundamental for the study of human gait. This paper presents a complete and systematic approach to deriving control system models for both the single support phase and the double support phase of a gait cycle. We consider a nine segment model in the sagittal plane. Emphasis is placed on two important aspects of the modeling process: dealing with the holonomic constraints and dealing with the ground reaction forces. We start with a raw model which is a direct result of Newton's law and which is subject to holonomic constraints. By characterizing the ground reaction forces, these constraints are eliminated to generate standard control system models for every possible situation in a gait cycle. These models are verified via both inverse dynamics and forward simulations on experimental data.
\end{abstract}

Keywords: gait model, holonomic constraints, ground reaction forces, forward simulation, inverse dynamics

\section{INTRODUCTION}

Walking appears to be a simple task for normal people. Yet it takes tremendous effort to build a machine that can barely make one full step, not to mention a robot that can walk as efficiently and gracefully as a human being. We also realize that walking is much more complicated than it appears to be when we make every effort to correct the walking strategies of disabled bodies so that they can walk like a normal person or with more efficiency. The apparent simplicity of walking disappears when one attempts to describe it as a scientific process: a dynamical control system.

Bipedal walking is a multidisciplinary subject that has been studied by a diverse group of clinicians and engineers including orthopedic surgeons, physical therapists, exercise physiologists, bioengineers, mechanical engineers and electrical engineers. Basically, two types of subjects are involved in the study of bipedal walking: the human and the bipedal robots. As a matter of fact, human walking and bipedal robot walking involve similar dynamical systems which can be studied in the same framework in control theory. Although the human body is much more complicated than a bipedal robot, the locomotion of a bipedal robot captures the fundamental properties of human walking, such as stability. The stability of bipedal walkers has been extensively studied in the robotics and control community [5], [8], [12].

It is now well accepted that the best way to understand walking is to model it as a dynamical system and to study its stability and performance in terms of input-output

\footnotetext{
${ }^{1}$ Department of Electrical and Computer Engineering

${ }^{2}$ Department of Orthopaedic Surgery

3 Department of Mechanical Engineering

$\dagger$ University of Virginia, Charlottesville, VA 22904-4743

Emails: \{th7f, zl5y, mfa2m, pea\}@virginia.edu
}

characteristics. There is a long history of modeling the bipedal walking and a wide range of models have been developed over the years with different levels of complexity. One end of the spectrum involves simple models with a minimal degree of freedom, such as ballistic and passive walking models (see, e.g., [1], [8], [14]). The other end of the spectrum consists of complex models which are actuated by muscle excitations (see, e.g., [2], [15], [18]). Models with different levels of complexity capture different aspects of walking characteristics [19] and attention has been paid to both the study with simple models and the development of complex models.

Models with medium level of complexity usually involve the feet, the lower legs (shanks), the upper legs (thighs) and the HAT (head-arm-trunk). These models are actuated by net joint moments and have been widely studied (see, e.g., [6], [7], [10], [16]). The more complicated models involving muscle excitations are actually built upon these models by attaching individual subsystems of muscle actuation at the original input points of joint moments or muscle forces. Therefore, it is important to study these models in depth and to have full understanding of the skeletal dynamics. Besides, these models have also been recognized to be useful in understanding the development of pathological gait (see, e.g., [7] and [19] and the references therein). For these reasons, our modeling effort in this paper will be focused on a nine segment bipedal walking system which is driven by the joint moments.

In the study of human walking, the gait cycle is divided into double support phases and single support (swing) phases. It is well known that the dynamics of the double support phase is much more difficult to describe than that of the single support phase. As a result, the majority of research results have been reported on the single support phase (see, e.g., [4], [7], [9], [11], [13]). However, since the double support phase generates most of the energy for forward progression and prepares the single support phase with initial position and velocity, simply explaining pathological gait from the swing phase may lead to incorrect conclusions and we may have to locate the origin of abnormality in the double support phase [4].

The difficulty in modeling the double support phase arises from the holonomic constraints imposed by the contact of both feet with the ground. Although this problem has been attempted by different researchers, the approaches are quite different and have not been unified yet. For example, in [6], the foot is modeled as a curved plantar surface which rolls on the ground but not slide, and in [2], the interaction is modeled with a series of springs and dampers on the soles of the feet. A more complicated ground contact 
model was used in [9]. In other literature, both feet are assumed fixed on the ground for simplicity (see, e.g., [3], [10]). In our preliminary study, we observed that neither of the feet is fixed on the ground during the double support phase. Although the relative motion between the feet and the ground is very small, the acceleration is considerable. Simply ignoring the acceleration would result in large errors in evaluating the kinetics and power transfer among the body segments. It would be significant and challenging to develop a relatively simple model that is able to account for the relative motion between the feet and the ground.

In this paper, we will develop a systematic approach to modeling a bipedal walker driven by the joint moments. We will start with a raw model which is directly obtained from Newton's law. This raw model has redundant variables in both the inputs and the states, and it is subject to holonomic constraint. The main effort of modeling will be devoted to the elimination of the redundant variables and to dealing with the holonomic constraint through characterizing the ground reaction forces. We will finally arrive at a set of models which are free of holonomic constraint for all possible situations in a gait cycle. These models have clean dynamical relationships between the inputs and the states, and will be validated with experimental data via both inverse dynamics and forward simulation.

\section{A WALKING SYSTEM, OBSERVATIONS AND ASSUMPTIONS}

\section{A. A nine segment bipedal walker}

We use a 9 segment linked body to study the motion in the sagittal plane (see Fig. 1). Each leg of the body has a thigh, a shank and a foot which consists of two segments: the toe and the hindfoot. The upper body is represented with a single segment. A full gait cycle has two single support phases and two double support phases. A single support phase starts when the toe of one foot leaves the ground (toe-off) and ends when the heel of the same foot touches the ground (heel-strike). A double support phase starts when the heel of one foot touches the ground and ends when the toe of the other foot leaves the ground. Since a normal walking is symmetric on both the left side and the right side, it suffices to study half of a gait cycle which starts with the toe-off of one foot and ends with the toe-off of another foot. During this half gait cycle, we call the leg that is not in touch with the ground in the single support phase the swing leg and the other leg the stance leg. The segments are numbered $1,2, \cdots$, starting from the toe of the stance leg. During the swing phase, the toe of the swing leg does not affect other segments much. During the double support phase, the foot in the front (or, of the swing leg) is almost straight. Because of these and for simplicity, we use a single segment to denote the foot of the swing leg, which should be the 8th segment according to our numbering.

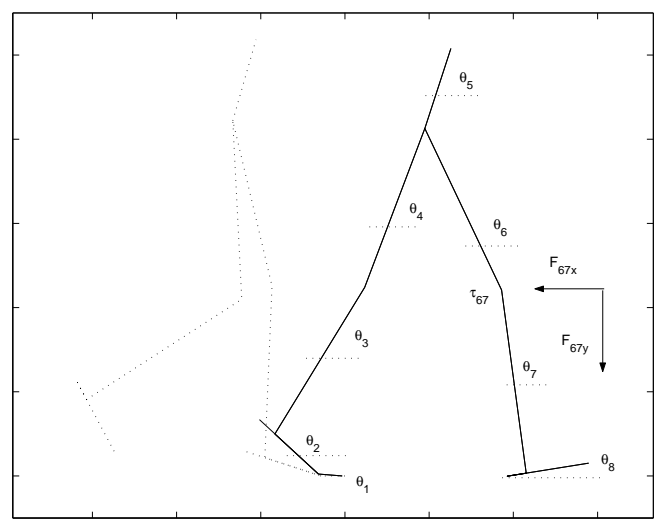

Fig. 1. A nine segment walker

\section{B. Observations and basic assumptions}

Traditionally, it is assumed that the foot of the stance leg is fixed on the ground during the single support phase and both feet are fixed during the double support phase. We also made such an assumption at the earlier stage of our research but later found out that this assumption would result in large discrepancy in forces and moments between the forward dynamics and the inverse dynamics. Careful examination of experimental data reveals that there are small relative motions between the feet and the ground.

\section{An observation: the relative motion}

From the experimental data that we collected on a group of tests on normal barefoot walking, we observed that there were relative motions between both feet and the ground during the double support phase. In the experiment, we placed a regular set of markers on the subject. The movement of the foot was usually measured with a marker at the heel (heel marker) and a marker at the joint between the toe segment and the hindfoot (toe marker). For better observation of the motion of the feet, we placed an additional marker on the tip of each big toe. The displacements of these markers are very small (within five centimeters) but the accelerations are considerable. If we ignore these accelerations, there would be significant differences between the real interaction forces and the interaction forces projected by the dynamics of the model. In other words, if we ignore these relative motions in the process of modeling, the resulting model would fail to describe the true dynamics of the walking system.

\section{Assumptions on the relative motion}

We use $x_{t}$ to denote the horizontal displacement of the tip of the toe of the stance leg and use $x_{h}$ to denote the horizontal displacement of the heel of the swing leg. We assume that, during the double support phase, the tip of the toe of the hind leg slides backward with a velocity $\dot{x}_{t}$ $\left(\dot{x}_{t} \leq 0\right)$ and the heel of the front leg slides forward with a velocity of $\dot{x}_{h}\left(\dot{x}_{h} \geq 0\right)$. These velocities might be zero for slow walking. We also assume that during the single support phase, there might be a backward velocity of the tip of the toe of the stance leg $\left(\dot{x}_{t} \leq 0\right)$. We observed such 
a possibility in some of the experiment on normal walking. It is likely that when the walking speed increases, the backward motion would increase and at the extreme case of running, this motion would be significant. This assumption has a potential to explain how fast walking transitions into running.

When there is a relative horizontal motion between the contacting surfaces, the horizontal interaction force can be categorized as the dynamic friction force. Let the horizontal ground reaction forces at the first segment and the 8th segment be $F_{g 1 x}$ and $F_{g 8 x}$, respectively, and let the vertical ground reaction forces at the first segment and the 8th segment be $F_{g 1 y}$ and $F_{g 8 y}$, respectively. When the foot slides on the ground, the horizontal force $F_{g 1 x}$ (or $F_{g 8 x}$ ) is a dynamic friction force. Its relation with the vertical force $F_{g 1 y}$ (or $F_{g 8 y}$ ) depends on the material of the ground and the footwear, and possibly, the sliding velocity. In general, the dynamic friction force is proportional to the vertical force. If the contacting surfaces are both rigid, then

$$
F_{g 1 x}=k_{t} F_{g 1 y}, \quad F_{g 8 x}=k_{h} F_{g 8 y},
$$

where $k_{t}$ and $k_{h}$ are constants. If the contacting surfaces are deformable, the friction force may also depend on the sliding velocity $\dot{x}_{t}$ and $\dot{x}_{h}$, i.e.,

$$
F_{g 1 x}=k_{t}\left(\dot{x}_{t}\right) F_{g 1 y}, \quad F_{g 8 x}=k_{h}\left(\dot{x}_{h}\right) F_{g 8 y} .
$$

It turns out that the relations $\left(F_{g 1 x}, F_{g 1 y}\right)$ and $\left(F_{g 8 x}, F_{g 8 y}\right)$ can be used to simplify the model. Our preliminary study indicates that these relations can be determined from experimental data.

\section{A RAW MODEL WITH HOLONOMIC CONSTRAINT}

Here we use the following notation to describe the kinematics and kinetics of the body segments:

- $\left(x_{i}, y_{i}\right)$ : the position of the center of mass of the $i$ th segment.

- $\theta_{i}$ : the angle between the $i$ th segment and the forward horizontal direction.

- $F_{j i x}, F_{j i y}$ : the total forces acted on the $i$ th segment from the $j$ th segment.

- $\tau_{i j}$ : the net moment at the joint between the $i$ th and the $j$ th segments.

The parameters of the body segments are listed as follows:

- $m_{i}$ : the mass of the $i$ th segment.

- $I_{i}$ : the moment of inertia of the $i$ th segment with respect to its center of mass.

Denote

$x=\left[\begin{array}{c}x_{1} \\ y_{1} \\ \vdots \\ x_{8} \\ y_{8}\end{array}\right], \theta=\left[\begin{array}{c}\theta_{1} \\ \vdots \\ \theta_{8}\end{array}\right], \dot{\theta}^{2}=\left[\begin{array}{c}\dot{\theta}_{1}^{2} \\ \vdots \\ \dot{\theta}_{8}^{2}\end{array}\right], c=\left[\begin{array}{c}\cos \theta_{1} \\ \vdots \\ \cos \theta_{8}\end{array}\right], s=\left[\begin{array}{c}\sin \theta_{1} \\ \vdots \\ \sin \theta_{8}\end{array}\right]$
Assume for simplicity that the ground reaction forces on the stance foot act at the tip of the toe. This is not the case when the entire toe part or the entire foot is in contact with the ground. When the whole segment of the toe is on the ground, the ground reaction force is distributed. To simplify the situation, we introduce a fictitious moment $\tau_{0}$ on the toe segment so that it produces an equivalent motion dynamics. This fictitious moment is manipulated by the muscles under the foot and hence can be considered as an input.

\section{The dynamical equations}

Denote

$$
\begin{aligned}
& F=\left[\begin{array}{lllllll}
F_{g 1 x} & F_{g 1 y} & \cdots & F_{87 x} & F_{87 y} & F_{g 8 x} & F_{g 8 y}
\end{array}\right]^{\mathrm{T}} \in \mathbf{R}^{18} \\
& \tau=\left[\begin{array}{llll}
\tau_{0} & \tau_{21} & \cdots & \tau_{87}
\end{array}\right]^{\mathrm{T}} \in \mathbf{R}^{8} \\
& M=\operatorname{diag}\left\{m_{1}, m_{1}, m_{2}, m_{2}, \cdots, m_{8}, m_{8}\right\} \in \mathbf{R}^{16 \times 16} \\
& G=\left[\begin{array}{lllllll}
0 & m_{1} g & 0 & m_{2} g & \cdots & 0 & m_{8} g
\end{array}\right]^{\mathrm{T}} \in \mathbf{R}^{16} \\
& I=\operatorname{diag}\left\{I_{1}, I_{2}, \cdots, I_{8}\right\} \in \mathbf{R}^{8 \times 8}
\end{aligned}
$$

The equations of motion are directly derived from Newton's law. We have

$$
\begin{aligned}
M \ddot{x} & =R F-G \\
I \ddot{\theta} & =W(\theta) F+B \tau
\end{aligned}
$$

where $R \in \mathbf{R}^{16 \times 18}, B \in \mathbf{R}^{8 \times 8}$ are constant matrices and $W(\theta) \in \mathbf{R}^{8 \times 18}$ is the moment arm matrix that depends on $\theta$. For simplicity, we omit the dependence of $W(\theta)$ on $\theta$ and simply write it as $W$, as will be done with other matrices depending on $\theta$.

\section{The constraints}

The centers of mass of the body segments are determined by the segment angles $(\theta)$ and the position of the distal end of the first segment $\left(x_{t}\right)$, i.e.,

$$
x=J_{1} s+J_{2} c+E x_{t}
$$

where $J_{1}, J_{2} \in \mathbf{R}^{16 \times 8}$ and $E \in \mathbf{R}^{16 \times 1}$ are constant matrices. We further have

$$
\ddot{x}=X_{1} \dot{\theta}^{2}+X_{2} \ddot{\theta}+E \ddot{x}_{t}
$$

where $X_{1}, X_{2} \in \mathbf{R}^{16 \times 8}$ depend on $\theta$,

$X_{1}=-J_{1} \operatorname{diag}(s)-J_{2} \operatorname{diag}(c), X_{2}=J_{1} \operatorname{diag}(c)-J_{2} \operatorname{diag}(s)$

For the double support phase, we need to deal with the constraint imposed by the contact of the feet of the ground. The position of the heel is determined as

$$
\left[\begin{array}{l}
x_{h} \\
y_{h}
\end{array}\right]=J_{3} s+J_{4} c+\left[\begin{array}{c}
x_{t} \\
0
\end{array}\right]
$$

where $J_{3}, J_{4} \in \mathbf{R}^{2 \times 8}$ are constant matrices. If the heel is fixed on the ground, then $\ddot{y}_{h} \equiv 0, \ddot{x}_{h} \equiv 0$. This constraint can be written as

$$
X_{3} \dot{\theta}^{2}+X_{4} \ddot{\theta}+\left[\begin{array}{c}
\ddot{x}_{t} \\
0
\end{array}\right]=0
$$


where $X_{3}, X_{4} \in \mathbf{R}^{2 \times 8}$ depend on $\theta$. If the heel slides on the ground, then $\ddot{y}_{h} \equiv 0$. This constraint can be written as

$$
X_{5} \dot{\theta}^{2}+X_{6} \ddot{\theta}=0
$$

where $X_{5}, X_{6} \in \mathbf{R}^{1 \times 8}$ depend on $\theta$.

The raw model

Putting together the equations of motion, the constraints and the relation between the ground reaction forces, we have the raw model as

$$
\begin{aligned}
M \ddot{x} & =R F-G \\
I \ddot{\theta} & =W F+B \tau \\
\ddot{x} & =\mid ! X_{1} \dot{\theta}^{2}+X_{2} \ddot{\theta}+E \ddot{x}_{t} \\
(6),(7), \text { or neither for single support, } & \\
F_{g 1 x} & =k_{t}\left(\dot{x}_{t}\right) F_{g 1 y} \text { if } \dot{x}_{t} \neq 0 \\
F_{g 8 x} & =k_{h}\left(\dot{x}_{h}\right) F_{g 8 y} \text { for double support, if } \dot{x}_{h} \neq 0 .(13)
\end{aligned}
$$

We call equations (8) - (13) the raw model because it is not clear which is the control input and which is the state, and the dynamical relationship between the input and the state is not explicit. In a standard control system

$$
\ddot{v}=f(v, \dot{v}, u),
$$

where $u$ is the control input and $(v, \dot{v})$ is the state, the trajectory of $v$ is completely determined by the input $u$ and the initial value of $(v, \dot{v})$.

The main objective of this paper is to obtain a control system model in the general form of (14) for the walking system. The first step is to choose the appropriate input and state. Traditionally, $\tau$ is chosen as the control input. It turns out that all the joint interaction forces and the ground reaction forces contained in the vector $F$ are detremined by the moment vector $\tau$. On the other hand, the state variables seem to include $x, \dot{x}, \theta, \dot{\theta}, x_{t}$ and $\dot{x}_{t}$ but there is also redundancy among them, since $x$ depends on $\theta$ and $x_{t}$. The possible state variables should be chosen among $\theta, \dot{\theta}, x_{t}$ and $\dot{x}_{t}$. Because of the holonomic constraints (6) and (7), there may still exist redundant states among $\theta, \dot{\theta}, x_{t}$ and $\dot{x}_{t}$. As we will see later, choosing of the state variables depends on different situtations of the gait cycle.

The next section is devoted to deriving a clean dynamical relationship between $\theta, x_{t}$ and $\tau$.

\section{THE CONTROL SYSTEM MODELS}

There are five possible cases during a gait cycle: two for the single support phase and three for the double support phase. These cases are classified in terms of the relative motion between the feet and the ground.

\section{A. The single support phase}

For the single support phase, the raw model is

$$
\begin{aligned}
M \ddot{x} & =R F-G \\
I \ddot{\theta} & =W F+B \tau \\
\ddot{x} & =X_{1} \dot{\theta}^{2}+X_{2} \ddot{\theta}+E \ddot{x}_{t} \\
F_{g 1 x} & =k_{t}\left(\dot{x}_{t}\right) F_{g 1 y} \quad \text { if } \dot{x}_{t} \neq 0
\end{aligned}
$$

Recall that $M \in \mathbf{R}^{16 \times 16}, R \in \mathbf{R}^{16 \times 18}, B \in \mathbf{R}^{8 \times 8}$, $I \in \mathbf{R}^{8 \times 8}, E \in \mathbf{R}^{16 \times 1}$ and $G \in \mathbf{R}^{16 \times 1}$ are constant matrices/vectors. The matrices $W \in \mathbf{R}^{8 \times 18}, X_{1}, X_{2} \in$ $\mathbf{R}^{16 \times 8}$ depend on the angle vector $\theta$.

During the single support phase, the last two elements of $F$ are zero, i.e., $F_{g 8 x}=F_{g 8 y}=0$. Let $F_{1} \in \mathbf{R}^{16}$ be formed by dropping $F_{g 8 x}$ and $F_{g 8 y}$ from $F$ and let $R_{1} \in$ $\mathbf{R}^{16 \times 16}$ and $W_{1} \in \mathbf{R}^{8 \times 16}$ be formed by dropping the last two columns from $R$ and $W$, respectively, then we have

$$
\begin{aligned}
M \ddot{x} & =R_{1} F_{1}-G \\
I \ddot{\theta} & =W_{1} F_{1}+B \tau
\end{aligned}
$$

There are two possible cases during the single support phase. In the first part of the phase, the tip of the toe is fixed on the ground, i.e., $\dot{x}_{t} \equiv \ddot{x}_{t} \equiv 0$. In the second part of the phase, the tip of the toe slides backward, i.e., $\dot{x}_{t}<0$. This second part might be absent for slow or normal walking. As the walking speed increases, the second part becomes more significant.

1) Case 1: $\dot{x}_{t} \equiv \ddot{x}_{t} \equiv 0$ : This is the simplest case in the whole gait cycle. The relation between $x$ and $\theta$ is

$$
\ddot{x}=X_{1} \dot{\theta}^{2}+X_{2} \ddot{\theta}
$$

From (19), we have

$$
F_{1}=R_{1}^{-1}\left(M X_{1} \dot{\theta}^{2}+M X_{2} \ddot{\theta}+G\right)
$$

It should be noted here that $R_{1}$ is invertible. Substituting (22) into (20), we have

$$
I \ddot{\theta}=W_{1} R_{1}^{-1}\left(M X_{1} \dot{\theta}^{2}+M X_{2} \ddot{\theta}+G\right)+B \tau
$$

By reorganizing the above equation, we obtain

$$
\ddot{\theta}=\left(I-W_{1} R_{1}^{-1} M X_{2}\right)^{-1}\left(W_{1} R_{1}^{-1}\left(M X_{1} \dot{\theta}^{2}+G\right)+B \tau\right)
$$

This is a clean dynamical relationship between the angle vector $\theta$ and the input $\tau$, which confirms that the trajectory of motion is driven by the joint moments. Moreover, all the joint reaction forces, including the ground reaction forces, are determined indirectly by the moments through (22) and (24). We can also use the above equations to perform inverse dynamics, i.e., to determine the forces and moments from the experimental kinematic data.

2) Case 2: $\dot{x}_{t}<0$ : In this case, we have an extra condition (18) in addition to (15)-(17). Also, $x_{t}$ is an extra degree of freedom besides all the segment angles. Hence the state vector should be $\left[\begin{array}{c}x_{t} \\ \theta\end{array}\right]$. The input vector is still $\tau$. In the sequal, we use $0_{m \times n}$ to denote an $m \times n$ matrix with all zero elements and use $I_{n \times n}$ to denote the $n \times n$ identity matrix.

Let $R_{1}$ be partitioned as $R_{1}=\left[\begin{array}{ll}R_{11} & R_{12}\end{array}\right]$ where $R_{11} \in$ $\mathbf{R}^{16 \times 1}$. Let $W_{1}$ be partitioned as $W_{1}=\left[\begin{array}{ll}W_{11} & W_{12}\end{array}\right]$ where 


$$
\begin{aligned}
& W_{11} \in \mathbf{R}^{8 \times 1} \text {. Let } T=\left[\begin{array}{ll}
1 & 0_{1 \times 14}
\end{array}\right] \text {. Denote } \\
& S_{1}=\left[\begin{array}{ll}
I_{15 \times 15} & 0_{15 \times 1}
\end{array}\right]\left[R_{11}\left(k\left(\dot{x}_{t}\right) T+R_{12}\right)-M E\right]^{-1} \\
& S_{2}=\left[\begin{array}{ll}
0_{1 \times 15} 1 & 1
\end{array}\right]\left[R_{11}\left(k\left(\dot{x}_{t}\right) T+R_{12}\right)-M E\right]^{-1} \\
& Q=\left[\begin{array}{ll}
1 & -S_{2} M X_{2} \\
0 & I-\left(W_{11} k_{t}\left(\dot{x}_{t}\right) T+W_{12}\right) S_{1} M X_{2}
\end{array}\right] .
\end{aligned}
$$

Then

$$
\begin{gathered}
{\left[\begin{array}{c}
\ddot{x}_{t} \\
\ddot{\theta}
\end{array}\right]=Q^{-1}\left[\begin{array}{c}
S_{2}\left(M X_{1} \dot{\theta}^{2}+G\right) \\
\left(W_{11} k_{t}\left(\dot{x}_{t}\right) T+W_{12}\right) S_{1}\left(M X_{1} \dot{\theta}^{2}+G\right)
\end{array}\right]} \\
+Q^{-1}\left[\begin{array}{c}
0 \\
B
\end{array}\right] \tau .
\end{gathered}
$$

\section{B. The double support phase}

1) Case 1: $\dot{x}_{t} \equiv \dot{x}_{h} \equiv 0$ : The raw model for this case is

$$
\begin{aligned}
M \ddot{x} & =R F-G \\
I \ddot{\theta} & =W F+B \tau \\
\ddot{x} & =X_{1} \dot{\theta}^{2}+X_{2} \ddot{\theta} \\
0 & =X_{3} \dot{\theta}^{2}+X_{4} \ddot{\theta}
\end{aligned}
$$

Because of the constraint (28), the degree of freedom of the system is two less than the number of segments. Recall that $X_{3}, X_{4} \in \mathbf{R}^{2 \times 8}$. In other words, two of the angles are determined by the remaining 6 angles.

Partition $R=\left[\begin{array}{ll}R_{1} & R_{2}\end{array}\right]$ with $R_{1} \in \mathbf{R}^{16 \times 16}$ and $R_{2} \in$ $\mathbf{R}^{16 \times 2}$. Partition $W=\left[\begin{array}{ll}W_{1} & W_{2}\end{array}\right]$ with $W_{1} \in \mathbf{R}^{8 \times 16}$ and $W_{2} \in \mathbf{R}^{8 \times 2}$. Let $S=\left(I-W_{1} R_{1}^{-1} M X_{2}\right)^{-1}$ and

$$
\begin{gathered}
P=X_{4} S\left(-W_{1} R_{1}^{-1} R_{2}+W_{2}\right) \\
P_{1}=S W_{1} R_{1}^{-1} M X_{1}+S\left(-W_{1} R_{1}^{-1} R_{2}+W_{2}\right) P^{-1} \\
\quad \times\left(-X_{3}-X_{4} S W_{1} R_{1}^{-1} M X_{1}\right) \\
P_{2}=S W_{1} R_{1}^{-1}-S\left(-W_{1} R_{1}^{-1} R_{2}+W_{2}\right) P^{-1} X_{4} S W_{1} R_{1}^{-1} \\
P_{3}=S B+S\left(-W_{1} R_{1}^{-1} R_{2}+W_{2}\right) P^{-1} X_{4} S B
\end{gathered}
$$

Then

$$
\ddot{\theta}=P_{1} \dot{\theta}^{2}+P_{2} G+P_{3} \tau
$$

For the system (29), the degree of freedom appears to be 8. But the special structure of $P_{1}, P_{2}$ and $P_{3}$ ensures that the holonomic constraint is automatically satisfied. Hence the actual degree of freedom is 6 .

2) Case 2: $\dot{x}_{t}<0, \dot{x}_{h}>0$ : The raw model for this case is

$$
\begin{aligned}
M \ddot{x} & =R F-G \\
I \ddot{\theta} & =W F+B \tau \\
\ddot{x} & =X_{1} \dot{\theta}^{2}+X_{2} \ddot{\theta}+E \ddot{x}_{t} \\
0 & =X_{5} \dot{\theta}^{2}+X_{6} \ddot{\theta} \\
F_{g 1 x} & =k_{t}\left(\dot{x}_{t}\right) F_{g 1 y} \\
F_{g 8 x} & =k_{h}\left(\dot{x}_{h}\right) F_{g 8 y}
\end{aligned}
$$

Here we have assumed different friction functions at the toe and the heel. The constraint (33) reduces the degree of freedom in the segment angles from 8 to 7 . In addition to the one degree of freedom at the toe of the hind leg $\left(x_{t}\right)$, the total degree of freedom is 8 .

Let $R_{1} \in \mathbf{R}^{16 \times 2}$ be formed by the first and the 17 th columns of $R$ and $R_{2} \in \mathbf{R}^{16 \times 16}$ be formed by the remaining columns of $R$. Let $W_{1}$ be formed by the first and the 17th columns of $W$ and let $W_{2}$ be formed by the remaining columns of $W$. Denote

$$
\begin{aligned}
& \left.T=\left[\begin{array}{cc}
k_{t}\left(\dot{x}_{t}\right) & 0_{1 \times 15} \\
{\left[0_{1 \times 15}\right.} & k_{h}\left(\dot{x}_{h}\right)
\end{array}\right]\right] \\
& S=\left(W_{1} T+W_{2}\right)\left(R_{1} T+R_{2}\right)^{-1} \\
& P=X_{6}\left(I-S M X_{2}\right)^{-1} S M E \\
& \Delta=\left(I-S M X_{2}\right)^{-1} \\
& P_{1}=\Delta\left(S M X_{1}-S M E P^{-1}\left(X_{5}+X_{6} \Delta S M X_{1}\right)\right) \\
& P_{2}=\Delta\left(S-S M E P^{-1} X_{6} \Delta S\right) \\
& P_{3}=\Delta\left(B-S M E P^{-1} X_{6} \Delta B\right)
\end{aligned}
$$

Then we have

$$
\ddot{\theta}=P_{1} \dot{\theta}^{2}+P_{2} G+P_{3} \tau
$$

The third case for the double support phase is $\dot{x}_{t}<0$, $\dot{x}_{h}=0$. The final model can be obtained similarly as case 2. It is omitted due to space limitation.

\section{EXPERIMENTAL VERIFICATION}

To validate our model, we collected kinematic data on a normal subject through a motion capture system (VICON612). The subject was instructed to walk on bare foot at a normal speed. All the segment angles and the joint centers are computed from the trajectories of a set of markers. The velocities and accelerations are then computed by digital differentiation. The ground reaction forces on both feet are also measured for verification. The segment parameters are estimated from the height and the weight of the subject by using the formulae in [16].

To validate the forward dynamics of the models, we feed the models with proper input signals and observe the resulting trajectories of the angles. As a first step, we feed the model with the moments directly from the calculation of the inverse dynamics. Then we alter the moments and observe how the changes affect the output. However, the process of forward simulation is much more complicated than it appears to be. For the single support phase, the complication results from the instability of the model. For the double support phase, arbitrarily altering the moments may produce meaningless outcome, such as unreasonable joint angles, even if the change is small.

\section{The single support phase}

During the single support phase, the body behaves like an inverted pendulum, which is unstable. The instability is caused by the gravity and will be weakened if we assume reduced gravitational acceleration.

To validate the model through simulation, we may assume a reduced gravitational acceleration and keep everything else in the model unchanged. Note that we should use 
the same gravitational acceleration for the inverse dynamics and the forward dynamics. Our simulation was carried out with Matlab's Simulink. We first assumed a normal
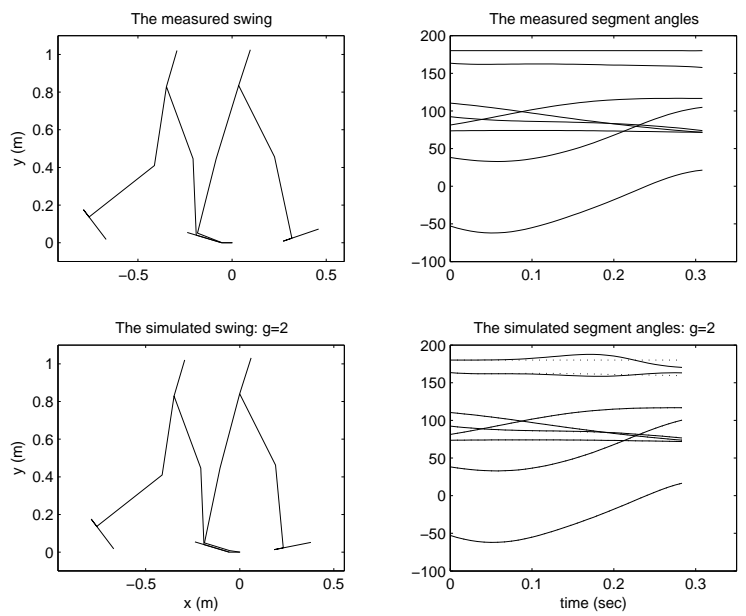

Fig. 2. Simulation under $g=2 m / s^{2}$.

gravitational acceleration $g=9.8 \mathrm{~m} / \mathrm{s}^{2}$ and computed the moments by using the inverse dynamics. By feeding the moments into the Simulink model, we observed that the trajectory of the simulated angles match the measured angles for the first 0.1 second but diverge quickly from them afterwards. We then reduced the gravity to $2 \mathrm{~m} / \mathrm{s}^{2}$ and repeated the procedure. A quite different result was then observed (see Fig. 2, where the dotted curves in the lower right plot are the angles (degree) from the experiment data and the solid curves are the angles from the forward simulation). If we reduce the gravity to 0 , then the simulated trajectory matches the measured trajectory perfectly, which validates our model.

The double support phase

In the first part of the double support phase, both feet slide backward or forward. By feeding the model with the moments calculated from the inverse dynamics, we obtained a simulated trajectory of the segment angles. They were exactly the same as the measured angles. This not only validated our model, but also showed that the double support model is much more stable than the single support model. Fig. 3 plots the trajectory of the angles on the rightside and the positions (initial and final) of the linked body segments on the left side.

\section{ACKNOWLEDGEMENTS}

This work was supported by the NIH grant R24 HD39631. We gratefully acknowledge Tim Franklin, Audra Jackson and Patty Payne for collecting the experimental data used in this research.

\section{REFERENCES}

[1] R. M. Alexander, "Simple models of human movement," Appl. Mech. Rev., 48, pp. 461-470, 1995.

[2] F. C. Anderson, A dynamic Optimization Solution for a Complete Cycle of Normal Gait. PhD Thesis. Univ. Texas, Austin. 1999.
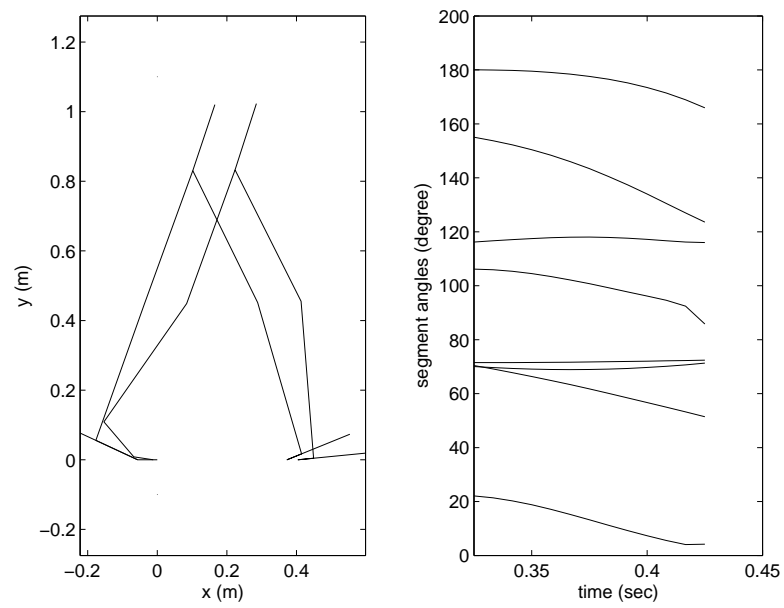

Fig. 3. Simulation with moments from the inverse dynamics

[3] A. Chemori and A. Loria, "Control of a planar five link underactuated biped robot on a complete walking cycle," Proc. of the 41th IEEE Conf. on Dec. \& Contr., pp. 2056-2061, Las Vegas, Nevada, 2002.

[4] S. R. Goldberg, S. Ounpuu and S. L. Delp, "The importance of swing-phase initial conditions in stiff-knee gait," J. of Biomechanics, 36, pp. 1111-1116, 2003.

[5] J. W. Grizzle, G. Abba and F. Plestan, "Asymptotically stable walking for biped robots: analysis via systems with impulse effect," IEEE Trans. Automat. Contr. 46, No. 1, pp. 51-64, 2001.

[6] M. S. Ju and J.M. Mansour, "Simulation of the double limb support phase of human gait," J. Biomech. Eng., 110, pp. 223-229, 1988.

[7] D. C. Kerrigan, R. S. Roth, P. O. Riley, "The modelling of adult spastic paretic stiff-legged gait swing period based on actual kinematic data," Gait and Posture, 7, pp. 117-124, 1998.

[8] A. D. Kuo, "A simple model of bipedal walking predicts the preferred speed-step length relationship," J. Biomech. Eng., 123, pp. 264-269, 2001.

[9] R. R. Neptune, I. C. Wright, A. J. van den Bogert, "A method for numerical simulation of single limb ground contact events: application to heel-toe running," Comp. Meth. of Biomechanical and Biomedical Eng., 3, pp. 321-334, 2000.

[10] M. G. Pandy and N. Berme, "A numerical method for simulating the dynamics of human walking," J. Biomech, 21, pp. 1043-1051, 1988.

[11] S. J. Piazza and S. L. Delp, "The influence of muscles on knee flexion during the swing phase of gait," J. Biomechanics, 29, pp. 723-733, 1996.

[12] D. Popovic and S. Jonic, "Control of bipedal locomotion assisted with functional electrical stimulation," Proc. of the American Control Conf., pp. 1238-1242, San Diego, 1999.

[13] P. O. Riley, "Torque action of two joint muscles in the swing period of stiff-legged gait: a forward dynamic model analysis," Journal of Biomech., 31, pp 835-840, 1998.

[14] M. W. Spong, R. Lozano and R. Mahony, "An almost linear biped," Proc. of the 39th IEEE Conf. on Dec. and Contr. pp. 4803-4808, 2000.

[15] D. G. Thelen, F. C. Anderson and S. L. Delp, "Generating dynamic simulations of movement using computed muscle control," J. of Biomech., 36, pp. 321-328, 2003.

[16] D. A. Winter, Biomechanics and Motor Control of Human Movement. New York: Wiley. 1990.

[17] E. R. Westervelt, J. W. Grizzle and C. Canudas de Wit, "Switching and PI control of walking motions of biped walkers," IEEE Trans. Automat. Contr., 48, No.2, pp. 308-312, 2003.

[18] G.T. Yamaguchi, Dynamic Modeling of Musculoskeletal Motion, Kluwer Academic Publishers, Boston, 2001.

[19] F. E. Zajac, R. R. Neptune and S. A. Kautz, "Biomechanics and muscle coordination of human walking: Part II: Lessons from dynamical simulations and clinical implications," Gait \& Posture, 17, 1-17, 2003. 